\title{
Remarks on the exact energy functional for fermions: an analysis using the Löwdin partitioning technique
}

\author{
Marc Caballero, ${ }^{\text {a,b }}$ Ibério de P. R. Moreira, ${ }^{\text {a,b }}$ Josep Maria Bofill ${ }^{\mathrm{a}, \mathrm{c}, *}$ \\ ${ }^{a}$ Institut de Química Teòrica i Computacional, Universitat de Barcelona, IQTCUB, \\ C/ Martí i Franquès 1, E-08028 Barcelona, Spain \\ bDepartament de Química Física, Universitat de Barcelona, \\ C/ Martí i Franquès 1, E-08028 Barcelona, Spain \\ 'Departament de Química Orgànica, Universitat de Barcelona, \\ C/ Martí i Franquès 1, E-08028 Barcelona, Spain \\ *Corresponding author. Email: jmbofill@ub.edu
}

(Version: August 29, 2013)

\begin{abstract}
.-
A comparison model based in the Löwdin partitioning technique is used to analyze the differences between the wave function and density functional models. This comparison model provides a tool to understand the structure of both theories and its discrepancies in terms of the subjacent mathematical structure and the variationality required for the energy functional. It is argued that the density functional theory can be compared to the wave-function theory. The wave-function theory provides an explicit form of the exact energy functional for a fermion system from the Full Configuration Interaction approach. The density functional theory can be seen as special cases of Löwdin function that do not satisfy all variational conditions on $\rho(\mathbf{r})$ and also on the $E_{X C}[\rho]$ term. This analysis shows that ignoring the restrictions imposed by the spin and space symmetry requirements of the solutions when making a variational calculation implies that the correlations expressed by the $\rho(\mathbf{r})$ function will be inconsistent with a $\gamma_{1}\left(\mathbf{r}_{1} ; \mathbf{r}_{1}{ }^{\prime}\right)$ function derivable from a spin and space symmetry adapted wave function $\Psi\left(\mathbf{r}_{1} s_{1}, \cdots, \mathbf{r}_{n} s_{n}\right)$, even for a closed-shell system. The comparison scheme also provides a new insight in order to achieve a consistent description of the molecular electronic structure of both ground and excited states. Some numerical results are reported.
\end{abstract}

Keywords: wave-function theory, density functional theory, Löwdin partition technique, variational methods, comparison model. 


\section{Introduction.}

The quantum many-electron system defined by $n$ electrons and $N$ nuclei in interaction is one of the central problems in chemistry and physics. The fundamental mathematical formulation of the non-relativistic $n$ electron problem is the time independent Schrödinger equation for this system and the corresponding exact solutions provide the essential quantum-mechanical description of each electronic state in terms of the different $n$ electron wave function $\Psi\left(\mathbf{r}_{1} s_{1}, \cdots, \mathbf{r}_{n} s_{n}\right)$.

For a system of $n$ electrons and $N$ nuclei in interaction, the time independent Schrödinger equation (in the Born-Oppenheimer approximation) can be written as a Rayleigh-Ritz quotient given by

$$
E[\Psi]=\frac{\langle\Psi|\hat{H}| \Psi\rangle}{\langle\Psi \mid \Psi\rangle}
$$

in which the Hamiltonian operator is defined as

$$
\hat{H}=\hat{T}+\hat{V}+\hat{W}
$$

with

$$
\begin{aligned}
& \hat{T}\left(\mathbf{r}_{1}, \cdots, \mathbf{r}_{n}\right)=-\frac{1}{2} \sum_{i=1}^{n} \nabla_{(i)}^{2} \\
& \hat{V}\left(\mathbf{r}_{1}, \cdots, \mathbf{r}_{n} ; \mathbf{R}_{1} ; \cdots ; \mathbf{R}_{N}\right)=\sum_{i=1}^{n} \sum_{I=1}^{N} \frac{-Z_{I}}{\left|\mathbf{R}_{I}-\mathbf{r}_{i}\right|}=\sum_{i=1}^{n} v\left(\mathbf{r}_{i} ; \mathbf{R}_{1} ; \cdots ; \mathbf{R}_{N}\right) \\
& \hat{W}\left(\mathbf{r}_{1}, \cdots, \mathbf{r}_{n}\right)=\sum_{i=1}^{n-1} \sum_{j=i+1}^{n} \frac{1}{\left|\mathbf{r}_{i}-\mathbf{r}_{j}\right|}=\sum_{i>j=1}^{n} \frac{1}{r_{i j}}
\end{aligned}
$$

In this expression, the first term stands for the electrons kinetic energy of the electrons, the second arises from the external potential $v\left(\mathbf{r}_{i} ; \mathbf{R}_{1} ; \cdots ; \mathbf{R}_{N}\right)$ generated by the nuclei and $1 / r_{i j}$ is the two electron interaction. This hamiltonian operator defines, along with its boundary conditions, an eliptic second order differential equation and the wave-function must satisfy some specific conditions to be an acceptable solution of Eq. (1), namely: a) $\Psi\left(\mathbf{r}_{1} s_{1}, \cdots, \mathbf{r}_{n} s_{n}\right)$ must be bounded and continuous, b) the partial derivatives with respect to spatial coordinates must be continuous, and c) the function $\left|\Psi\left(\mathbf{r}_{1} s_{1}, \cdots, \mathbf{r}_{n} s_{n}\right)\right|^{2}$ must be integrable. Since the non-relativistic many-electron Hamiltonian does not act on spin coordinates, anti-symmetry and spin restrictions must be imposed ad hoc to restrict the solutions (i.e. the wave functions $\Psi\left(\mathbf{r}_{1} s_{1}, \cdots, \mathbf{r}_{n} s_{n}\right)$ ) to 
the set of functions that satisfy the Pauli principle and spin symmetry requirements of the quantum mechanical state of the system.

In the wave-function theory (WFT) formalism, the most compact expression for the energy of the $n$-electron system in the field of $N$ fixed nuclei can be written as

$$
\begin{aligned}
E\left[\gamma_{1}, \gamma_{2}\right]= & -\frac{1}{2} \int_{\mathbf{r}_{1}=\mathbf{r}_{1}}\left[\nabla \cdot \nabla^{T} \gamma_{1}\left(\mathbf{r}_{1} ; \mathbf{r}_{1}^{\prime}\right)\right] d \mathbf{r}_{1}+ \\
& +\int_{\mathbf{r}_{1}} v\left(\mathbf{r}_{1} ; \mathbf{R}_{1} ; \cdots ; \mathbf{R}_{N}\right) \gamma_{1}\left(\mathbf{r}_{1}\right) d \mathbf{r}_{1}+ \\
& +\iint_{\mathbf{r}_{2}} \frac{\gamma_{2}\left(\mathbf{r}_{1}, \mathbf{r}_{2}\right)}{r_{12}} d \mathbf{r}_{1} d \mathbf{r}_{2}
\end{aligned}
$$

in which the many electron quantities $\gamma_{1}\left(\mathbf{r}_{1}\right)$ and $\gamma_{2}\left(\mathbf{r}_{1}, \mathbf{r}_{2}\right)$ are the diagonal elements, $\gamma_{1}\left(\mathbf{r}_{1}\right)=\gamma_{1}\left(\mathbf{r}_{1} ; \mathbf{r}_{1}\right)$ and $\gamma_{2}\left(\mathbf{r}_{1}, \mathbf{r}_{2}\right)=\gamma_{2}\left(\mathbf{r}_{1}, \mathbf{r}_{2} ; \mathbf{r}_{1}, \mathbf{r}_{2}\right)$, of the spinless one- and two-electron density matrices, respectively [1-3] The function $\gamma_{1}\left(\mathbf{r}_{1}\right)=\gamma_{1}\left(\mathbf{r}_{1}, \mathbf{r}_{1}\right)$ corresponds to an observable, the one electron density $\rho(\mathbf{r})$, and is commonly used in electronic structure theory. In WFT, large efforts are devoted to obtain accurate prediction of the energy of a given system in a given electronic state using a reasonable estimate of $\Psi\left(\mathbf{r}_{1} s_{1}, \cdots, \mathbf{r}_{n} s_{n}\right)$. It is customary to expand $\Psi\left(\mathbf{r}_{1} s_{1}, \cdots, \mathbf{r}_{n} s_{n}\right)$ in a known basis set and to find the expansion coefficients using the variational method and with all necessary and sufficient constraints (spin and space symmetries) to prevent the variational collapse [4]. This mathematical requirement is essential to avoid to converge to a solution with no physical meaning. This is the basis of the so-called Full Configuration Interaction (FCI) method which provides the exact solution for the energy functional of the electronic system defined in Eq. (3) in a given basis set. Indeed, for a finite basis set this is the exact solution and has been extensively used as a benchmark for quantum chemical methods $[5,,, 9]$.

Density Functional Theory $(D F T)$ propose a different approach which aims to replace both $\gamma_{1}\left(\mathbf{r}_{1}, \mathbf{r}_{1}{ }^{\prime}\right)$ and $\gamma_{2}\left(\mathbf{r}_{1}, \mathbf{r}_{2}\right)$ in Eq. (3) by the one-electron density, $\gamma_{1}\left(\mathbf{r}_{1}\right)$. For the ground state, this wish is justified by the celebrated Hohenberg-Kohn theorems $(H K)$, which state that the exact ground state total energy of any many-electron system is given by a universal, unknown, functional of the electron density only [10]. Rigorously speaking, only the second term of the right hand side part of Eq. (3) is an explicit functional of the diagonal one-electron density matrix, $\gamma_{1}\left(\mathbf{r}_{1}\right)$. The first term, which 
corresponds to the kinetic energy, is an explicit functional of the complete one-electron density matrix, $\gamma_{1}\left(\mathbf{r}_{1} ; \mathbf{r}_{1}{ }^{\prime}\right)$. The major contribution to the electron-electron term comes from the classical electrostatic 'self energy' of the charge distribution, which is also an explicit functional of the diagonal one-electron density matrix $\left[{ }^{1}\right]$. The remaining contribution of the electron-electron term is an explicit functional of $\gamma_{2}\left(\mathbf{r}_{1}, \mathbf{r}_{2}\right)$. It is important to stress the well known fact that the two-electron density $\gamma_{2}\left(\mathbf{r}_{1}, \mathbf{r}_{2}\right)$ cannot be factorized in terms of $\gamma_{1}\left(\mathbf{r}_{1} ; \mathbf{r}_{1}{ }^{\prime}\right)$ in the expression of the exact energy of the exact ground-state, even for a closed-shell system. In $D F T$, this and the non-diagonal part of the electron kinetic energy term are usually added into a so-called 'exchangecorrelation' functional which also depends on the one-electron density only $\left(E_{X C}[\rho]\right)$. The definition of $E_{X C}[\rho]$ is the basis for the practical use of $D F T$. Since $E_{X C}[\rho]$ is a functional of the density, it is possible to define a universal functional which is derivable from the one-electron density itself and without reference to the external potential $v\left(\mathbf{r}_{i} ; \mathbf{R}_{1} ; \cdots ; \mathbf{R}_{N}\right)$. Hence, DFT offers a way to eliminate the connection with the $n$ electron wave function working in terms of the density function $\rho(\boldsymbol{r})$ alone. In addition, since the first $H K$ theorem states that there exists a one-to-one mapping between the external potential $v\left(\mathbf{r}_{i} ; \mathbf{R}_{1} ; \cdots ; \mathbf{R}_{N}\right)$, the particle density $\gamma_{1}\left(\mathbf{r}_{1}\right)$ (or $\left.\rho(\mathbf{r})\right)$ it follows that $\rho(\mathbf{r})$ determines the exact non relativistic Hamiltonian (Eq. (1)) and hence one may, incorrectly, claim that $\rho(\mathbf{r})$ does also determine the ground state wave function $\Psi\left(\mathbf{r}_{1} s_{1}, \cdots, \mathbf{r}_{n} s_{n}\right)$. However, one must advert that, using the exact non-relativistic FCI wave function, information regarding $\gamma_{1}\left(\mathbf{r}_{1}\right)$ and $\gamma_{2}\left(\mathbf{r}_{1}, \mathbf{r}_{2}\right)$ is required to reconstruct the energy of the system provided that the spin is introduced ad hoc to fulfill the Pauli principle.

It is interesting to reformulate the exact energy functional expressed in Eq. (3) to provide a general expression to compare the WFT and DFT theories using a common language. To this end, following McWeeny $\left[{ }^{1}\right]$ one should reformulate $D F T$ extending Levy's constrained search [11] to ensure not only that the variational procedure leads to a $\gamma_{1}\left(\mathbf{r}_{1}\right)$ which derives from some wave function $\Psi\left(\mathbf{r}_{1} s_{1}, \cdots, \mathbf{r}_{n} s_{n}\right)$ (the $N$-representability problem $)^{4}$ but also that $\Psi\left(\mathbf{r}_{1} s_{1}, \cdots, \mathbf{r}_{n} s_{n}\right)$ belongs to the appropriate irreducible representation of the spin permutation group $S_{n}$ (the Pauli principle). The above proposition, corresponding to Eq. (1), can be written in a mathematical form by rewriting Eq. (3) as 


$$
\begin{aligned}
E\left[\gamma_{1}, \gamma_{2}\right]=\min _{\substack{\rho \rightarrow \gamma_{1} \text { derived } \\
\text { from } \Psi \in S_{n}}} & \left\{-\frac{1}{2} \int_{\mathbf{r}_{1}=\mathbf{r}_{1}}\left[\nabla \cdot \nabla^{T} \gamma_{1}\left(\mathbf{r}_{1} ; \mathbf{r}_{1}^{\prime}\right)\right] d \mathbf{r}_{1}+\right. \\
& +\int_{\mathbf{r}_{1}} v\left(\mathbf{r}_{1} ; \mathbf{R}_{1} ; \cdots ; \mathbf{R}_{N}\right) \gamma_{1}\left(\mathbf{r}_{1}\right) d \mathbf{r}_{1}+ \\
& \left.+\frac{1}{2} \iint_{\mathbf{r}_{1} \mathbf{r}_{2}=\mathbf{r}_{2}^{\prime}} \frac{\gamma_{1}\left(\mathbf{r}_{1}\right)\left(1-P_{12}\right) \gamma_{1}\left(\mathbf{r}_{2} ; \mathbf{r}_{2}^{\prime}\right)}{r_{12}} d \mathbf{r}_{1} d \mathbf{r}_{2}\right\}+ \\
& +\min _{\substack{\gamma_{2} \text { derived } \\
\text { from } \Psi \in S_{n}}}\left\{E_{\text {Correlation }}\left[\gamma_{2}\left(\mathbf{r}_{1}, \mathbf{r}_{2}\right)\right]\right\}
\end{aligned}
$$

which clearly shows the one-to-one relation between the one-electron density matrix, $\gamma_{1}\left(\mathbf{r}_{1} ; \mathbf{r}_{1}{ }^{\prime}\right)$, and the main part of the energy $E$ and the explicit dependence of the electronelectron correlation on $\gamma_{2}\left(\mathbf{r}_{1}, \mathbf{r}_{2}\right)$. Notice that if $E_{\text {Correlation }}\left[\gamma_{2}\left(\mathbf{r}_{1}, \mathbf{r}_{2}\right)\right]$ in Eq. (4) is forced to be zero one obtains another form of the well-known Hartree-Fock energy expression. In DFT, according to the $H K$ theorem, ultimately $E_{\text {Correlation }}\left[\gamma_{2}\left(\mathbf{r}_{1}, \mathbf{r}_{2}\right)\right]$ is also assumed as a function of the one electron density only and, if this is written in terms of the electron density, one obtains the Kohn-Sham equations [12] provided the non-diagonal terms of the kinetic energy and those arising from the permutation operator are all included in $E_{X C}[\rho]$. In the Hartree-Fock method, the energy is obtained trough a variational iterative procedure which involves the non-local Fock operators [13]. In DFT the variational problem possesses the same mathematical structure of the Hartree-Fock problem and it can be also solved iteratively leading to the Khon-Sham $(K S)$ equations [12]. The current implementation of DFT based methods differ in the particular way to model the unknown $E_{X C}[\rho]$ term. Taking into account this comparison and within the language of the DFT model, Eq. (4) can be written in a more compact form as

$$
E[\rho]=E_{K S}[\rho]+E_{X C}[\rho]
$$

where $E_{K S}[\rho]$ is the Khon-Sham energy and accounts for the kinetic, nuclear potential and Coulomb terms, whereas $E_{X C}[\rho]$ accounts for the exchange term plus correlation energy. This correlation energy is the extra energy term not contained in the $E_{K S}[\rho]$ plus exchange terms.

In this work, we analyze the mathematical structure of the exact energy functional for fermions derived from FCI by extending the analysis that we introduced in a previous work [14] where we have established a comparison scheme between the WFT and DFT methods to compare energy functionals defined by the same external potential 
$v\left(\mathbf{r}_{i} ; \mathbf{R}_{1} ; \cdots ; \mathbf{R}_{N}\right)$ and using the same basis set to describe the system of $n$ electrons. To this end, we use the Löwdin partitioning technique $[15,16]$ constructed using the exact non-relativistic FCI solution of Eq. (1) using different sets of basis functions (orbitals) defined in a minimal basis set. We apply this analysis to simple and well defined molecular systems, namely, $\mathrm{H}_{2} \mathrm{O}, \mathrm{SH}_{2}, \mathrm{NH}_{3}, \mathrm{CH}_{4}, \mathrm{NH}_{4}^{+}$(closed shell singlets) and $\mathrm{CH}_{2}$ and $\mathrm{O}_{2}$ (triplet ground state) to provide some numerical results that show de inherent structure of the energy functional and the dependence of the Löwdin function with respect to the orbitals used to construct the $F C I$ space.

\section{The Löwdin partitioning technique and its related function.}

In a previous work [14] we have proposed a comparison scheme to establish an equivalence between the WFT and DFT methods. The aim of this comparison model is to compare different energy functionals defined by the same external potential $v\left(\mathbf{r}_{i} ; \mathbf{R}_{1} ;\right.$ $\left.\cdots ; \mathbf{R}_{N}\right)$ generated by the $N$ fixed nuclei and using the same basis set to describe the system of $n$ electrons. To this end, we split the energy functional of the system as

$$
E=E_{\text {ref }}+E_{\text {Corr }} \text {. }
$$

By exploring simple forms of the component functionals it is possible to establish some equivalences in the subjacent mathematical structure between different energy functionals. We emphasize that this equivalence does not mean equality and our intention is to provide a comparison criterion for WFT and DFT based energy functionals. For this purpose we use the Löwdin partitioning technique of a secular equation $[15,16]$ applied to the $F C I$ electronic Hamiltonian in a given basis set and number of electrons, $n$, to solve the time independent Schrödinger equation given in Eq. (1). We split the $F C I$ electronic Hamiltonian secular equation of dimension $K$ through $I$ and $I I$ subspaces $\left(K=K_{I}+K_{I I}\right)$ as follows:

$$
\left(\begin{array}{cc}
\mathbf{H}_{I, I} & \mathbf{H}_{I, I I} \\
\mathbf{H}_{I I, I} & \mathbf{H}_{I I, I I}
\end{array}\right)\left(\begin{array}{c}
\mathbf{c}_{I}^{(i)} \\
\mathbf{c}_{I I}^{(i)}
\end{array}\right)=E_{i}\left(\begin{array}{c}
\mathbf{c}_{I}^{(i)} \\
\mathbf{c}_{I I}^{(i)}
\end{array}\right)
$$

For any eigenvalue, $E_{i}$, for which the components of the corresponding eigenvector $\left(\mathbf{c}^{(i)}\right)^{T}=\left(\mathbf{c}_{I}^{(i)} \mathbf{c}_{I I}^{(i)}\right)^{T} \neq\left(\mathbf{0}_{I} \mathbf{0}_{I I}\right)^{T}$, being $\mathbf{0}_{I}$ and $\mathbf{0}_{I I}$ the zero vectors of the subspaces $I$ and $I I$ respectively, the solutions of the secular equation given in Eq. (7) are equivalent to the solutions of the partitioned secular equation 


$$
\left[\mathbf{H}_{I, I}-\mathbf{H}_{I, I I}\left(\mathbf{H}_{I I, I I}-E_{i} \mathbf{I}_{I I, I I}\right)^{-1} \mathbf{H}_{I I, I}\right] \mathbf{c}_{I}^{(i)}=E_{i} \mathbf{c}_{I}^{(i)}
$$

where $\mathbf{I}_{I I, I I}$ is the identity matrix in the $I I$ subspace. For simplicity, in the present analysis we take the subspace $I$ of dimension one with $\mathbf{H}_{I, I}=H_{11}=E_{\text {ref }}$, i.e.: only a Configuration State Function (CSF) defines this subspace to represent singlet or triplet electronic states of representative systems. The rest of the CSFs of the FCI space provide the basis of the $I I$ subspace. Now we define the Löwdin function $f(E)$ which can be seen as the "eigenvalue" of the one-dimensional matrix $\left[\mathbf{H}_{I, I}-\mathbf{H}_{I, I I}\left(\mathbf{H}_{I I, I I}-E \mathbf{I}_{I I, I I}\right)^{\text {- }}\right.$ ${ }^{l} \mathbf{H}_{I I, I} I$, more explicitly, $f(E)$, can be written as a "Rayleigh-Ritz" quotient of this one dimensional matrix with an one-dimensional vector, say $\mathbf{d}$, with a coefficient $d=1$ due to normalization,

$$
f(E)=\mathbf{d}^{T}\left[\mathbf{H}_{I, I}-\mathbf{H}_{I, I I}\left(\mathbf{H}_{I I, I I}-E \mathbf{I}_{I I, I I}\right)^{-l} \mathbf{H}_{I I, I}\right] \mathbf{d} \quad \mathbf{d}^{T} \mathbf{d}=1 \quad \mathbf{d}=d=1
$$

Notice that in the present case $\mathbf{H}_{I, I I}=\left(\mathbf{H}_{I I, I}\right)^{T}$ is a vector of dimension $K-1, \mathbf{H}_{I I, I I}$ is a matrix of dimension $(K-1) \mathbf{x}(K-1)$, and finally $\mathbf{H}_{I, I}$ is an element of the $\mathbf{H}$ matrix. The domain of $E$ is $E \in(-\infty, \infty)$. The set of $K$ values of the Löwdin function such that $f(E)$ takes the value of $E, f(E)=E=E_{i}$ corresponds to the set of $\mathrm{K}$ eigenvalues of the secular equation given in Eq. (7). In this case $\mathbf{d}=\mathbf{c}_{I}^{(i)}\left[\left(\mathbf{c}_{I}^{(i)}\right)^{T}\left(\mathbf{c}_{I}^{(i)}\right)\right]^{-1 / 2}$ if $\mathbf{c}^{(i)}$ is a normalized vector. The function $f(E)$ is a non-increasing function of $E$. When the function $f(E)$ is represented in front of $E$, the horizontal asymptote of $f(E)$ tends to the value of the matrix element, $\mathbf{H}_{I, I}=H_{11}$ i.e.: $\lim _{E \rightarrow \pm \infty} f(E)=\mathbf{H}_{I, I}=E_{r e f}$. This limit coincides with the Hartree-Fock energy if the FCI electronic Hamiltonian has been constructed using the set of orbitals that makes stationary the one-CSF energy functional, $E_{r e f}\left[\gamma_{1}\right]=E_{H F}\left[\gamma_{1}\right]=$ $\mathbf{H}_{I, I}\left[\gamma_{1}\right]$. However, the remaining term in Eq. (9), namely, the $\mathbf{H}_{I, I I}=\left(\mathbf{H}_{I I, I}\right)^{T}$ vector and $\mathbf{H}_{I I, I I}$ matrix, are very complex functions that can be expressed in terms of the oneelectron components of the two-electron density matrix. Hence, at the point where $f(E)$ $=E=E_{i}$ the variational condition required in Eq. (7) for the eigenstate $i$ is satisfied, the Löwdin function given in Eq. (9) defines an energy functional that can be expressed more explicitly as $E\left[\gamma_{1}, E\right]$ taking into account that the $\mathbf{H}_{I, I I}=\left(\mathbf{H}_{I I, I}\right)^{T}$ vector and $\mathbf{H}_{I, I I}$ matrix can be expressed in terms of the one-electron components of the two-electron density matrix $\gamma_{2}\left(\mathbf{r}_{1}, \mathbf{r}_{2} ; \mathbf{r}_{1}{ }^{\prime}, \mathbf{r}_{2}{ }^{\prime}\right)$. The details of these relations are described in the next section. 


\section{Energy functionals from the Löwdin partition function}

At the point where $f(E)=E=E_{i}$ the variational condition required in the $F C I$ equation for the eigenstate $i$ is satisfied, the Löwdin function defines an energy functional that can be expressed explicitly as

$$
E\left[\gamma_{1}, E\right]=f\left[\mathbf{H}_{I, I}\left(\gamma_{1}\right), \mathbf{H}_{I, I I}, \mathbf{H}_{I I, I I}, E\right]=f\left[E_{H F}\left(\gamma_{1}\right), \mathbf{H}_{I, I I}, \mathbf{H}_{I I, I I}, E\right]
$$

and represents the simplest expression for the energy functional that can be derived from the $F C I$ that satisfies all necessary and sufficient variational conditions to be a solution of the Non Relativistic-Time Independent-Schrödinger Equation for $n$ electrons and $N$ nuclei defined by Eq. (1) in a given basis set. The simplest form of the energy functional in the previous expression, namely $E\left[\gamma_{1}, E\right]$, can be derived as follows. Following Roos et al. [17] the $\mathbf{H}_{I, I I}=\left(\mathbf{H}_{I I, I}\right)^{T}$ vector and the $\mathbf{H}_{I I, I I}$ matrix are functions of the one-electron components of the two-electron density matrix (a fourth rank tensor) for electronic state $L$ given by

$$
\left({ }^{L} \gamma_{2}\right)_{i j, k l}=\sum_{I, J, K}\left[c_{I}^{L}\left\langle I\left|E_{i j}\right| K\right\rangle\left\langle K\left|E_{k l}\right| J\right\rangle c_{J}^{L}-\delta_{j k} c_{I}^{L}\left\langle I\left|E_{i l}\right| J\right\rangle c_{J}^{L}\right]
$$

where $E_{i j}=a_{i \alpha}^{+} a_{j \alpha}^{-}+a_{i \beta}^{+} a_{j \beta}^{-}$and $a_{i \alpha}^{+}$(resp. $\left.a_{j \alpha}^{-}\right)$is the creation operator of an alpha electron in orbital $i$ (resp. the anihilation operator of an alpha electron in orbital $j$ ) and $I, J$, and $K$ are $C S F$ s of the $F C I$ space defined in a given basis set. The term

$$
\left(\gamma_{2}^{I J}\right)_{i j, k l}=\sum_{K}\left[\left\langle I\left|E_{i j}\right| K\right\rangle\left\langle K\left|E_{k l}\right| J\right\rangle-\delta_{j k}\left\langle I\left|E_{i l}\right| J\right\rangle\right]
$$

is the $i j, k l$ component of the two-electron density matrix formed by the $I, J C S F \mathrm{~s}$. Now, defining the one electron density matrix (a rank two tensor) for state $L$ as

$$
{ }^{L} \gamma_{1}=\sum_{I, J} c_{I}^{L}\left\langle I\left|E_{i j}\right| J\right\rangle c_{J}^{L}=\sum_{I, J} c_{I}^{L}\left(\gamma_{1}^{I J}\right)_{i j} c_{J}^{L}
$$

where

$$
\left(\gamma_{1}^{I J}\right)_{i j}=\left\langle I\left|E_{i j}\right| J\right\rangle
$$


is the $i j$ component of the one-electron density matrix formed by the $I, J C S F \mathrm{~s}$, the $i j, k l$ component of the two-electron density matrix formed by the $I, J C S F$ s given in Eq. (12) can be rewritten as

$$
\left(\gamma_{2}^{I J}\right)_{i j, k l}=F\left(\left\{\left(\gamma_{1}^{I J}\right)_{k l}\right\}\right)=\sum_{K}\left[\left(\gamma_{1}^{I K}\right)_{i j}\left(\gamma_{1}^{K J}\right)_{k l}-\delta_{j k}\left(\gamma_{1}^{I J}\right)_{i l}\right]
$$

Using the above definitions the second order reduced density matrix element $i j, k l$ for the electronic state $L$ can be rewritten as

$$
\left({ }^{L} \gamma_{2}\right)_{i j, k l}=\sum_{I, J} c_{I}^{L}\left(\gamma_{2}^{I J}\right)_{i j, k l} c_{J}^{L}=\sum_{I, J, K}\left[c_{I}^{L}\left(\gamma_{1}^{I K}\right)_{i j}\left(\gamma_{1}^{K J}\right)_{k l} c_{J}^{L}-\delta_{j k} c_{I}^{L}\left(\gamma_{1}^{I J}\right)_{i l} c_{J}^{L}\right]
$$

With these premises, the simplest expression for the energy functional for a fermion system that takes into account all necessary and sufficient variational conditions (and antisymmetry) can be reduced to

$$
f(E)=f\left[\mathbf{H}_{I, I}\left(\gamma_{1}\right), \mathbf{H}_{I, I I}, \mathbf{H}_{I I, I I}, E\right] .
$$

This energy functional corresponds to the exact energy derived from the $F C I$ and explicitly depends on the one electron density matrix, the set of component one-electron density matrices defined in Eq. (14) necessary to build the two-electron density matrix, and $E$.

At the value $E=0$ the above function $f(E)$ given in Eq. (17) reduces to the form

$$
f(0)=f\left[\mathbf{H}_{I, I}\left(\gamma_{1}\right), \mathbf{H}_{I, I I}, \mathbf{H}_{I I, I I}, 0\right]=\mathbf{d}^{T}\left[\mathbf{H}_{I, I}-\mathbf{H}_{I, I I}\left(\mathbf{H}_{I I, I I}\right)^{-1} \mathbf{H}_{I I, I}\right] \mathbf{d}
$$

with $\mathbf{d}=d=1$, and defines a parametric functional of $\gamma_{1}$ through $\mathbf{H}_{I, I}$ and the set $\left\{\gamma_{1}^{I J}\right\}$ through $-\mathbf{H}_{I, I I}\left(\mathbf{H}_{I I, I I}\right)^{-1} \mathbf{H}_{I I, I^{*}}$ This expression resembles Eq. (5) provided that it constructed using the set of orbitals that optimizes the functional $E_{H F}=\mathbf{H}_{I, I}$ and adding the kinetic and the exchange terms of $\mathbf{H}_{I, I}$ to the second term, namely, $-\mathbf{H}_{I, I I}\left(\mathbf{H}_{I I, I I}\right)^{-1} \mathbf{H}_{I I, I}$. The dependence of the set of orbitals is analyzed below although the FCI energy is invariant with respect to any unitary transformation of orbitals [13]. The resulting expression for this second term resembles to the $E_{X C}[\rho]$ functional in $D F T$. Notice that the $E_{X C}[\rho]$ functional does not depend on the energy itself. With this consideration the function $f(E)=f\left[\mathbf{H}_{I, I}\left[\gamma_{I}\right], \mathbf{H}_{\mathrm{I}, \mathrm{II}}, \mathbf{H}_{\mathrm{IIIII}}, E\right]$ at $E=0$ can be used to compare the WFT with the energy functional, $E[\rho]$, of the $D F T$. If one represents or plots $f(E)$ in front of $E$, the 
energy of any DFT method should be located on the vertical axis $f(0)$. The reason, as noted above, is because the $E_{X C}[\rho]$ of any $D F T$ method is equivalent to the second term of the $f(0)$ Löwdin function where in this case $E$ does not appear explicitly in the function as occurs in DFT. So far it seems the most licit and appropriated way to compare both models, namely, the WFT, also so-called ab initio methods, and the DFT. The functions representing both theories, namely, $E[\rho]$ in Eq. (5) and the Löwdin function $f(E)$ at $E=0$ from Eq. (9) are equivalent if they are build using the same basis set with the same external potential $v\left(\mathbf{r}_{i} ; \mathbf{R}_{1} ; \cdots ; \mathbf{R}_{N}\right)$, the same number of electrons, $n$, and in addition in DFT the set of orbitals is the $K S$ while in WFT is $H F$. With these two premises the comparison is well established and permitted.

\section{Numerical analysis of simple molecular systems.}

In this section, we compute the above described Löwdin partitioning function (cf. Eq. (9)) for representative, simple, stable and well-defined molecular systems in their electronic ground state and analyze the behavior of several $E_{X C}[\rho]$ functionals by comparison. For simplicity, we have taken the optimized geometry using the singles and doubles $C I$ (SDCI) wave function over the RHF (ROHF for triplet states) single $C S F$ reference and the $6-31++G^{* *}$ basis set. In all cases, this geometry has been used to extract all the roots of the $F C I$ expansion in a minimal $S T O-3 G$ basis set using the Graphical Unitary Group Approach (GUGA) algorithm [18] and the molecular symmetry except for degenerate groups, where a suitable non-degenerate subgroup has been considered. To this end, different sets of orbitals have been used to solve the $F C I$ problem and construct the Löwdin function for all the molecular systems considered. The FCI eigenvalues and eigenvectors have been used to construct the Löwdin function described above. The Kohn-Sham DFT energies using the same CISD/6-31++G** optimized geometry and the $S T O-3 G$ basis set have been calculated using representative local, $G G A$, meta- $G G A$ and hybrid $E_{X C}[\rho]$ functionals. $C I$ calculations have been carried out using the GAMESS 2012 code $[19,20]$ whereas the DFT calculations have been carried out using the GAMESS 2012 and the Gaussian09 code [21].

Regarding the molecular geometry of the systems used in the reported calculations, for $\mathrm{H}_{2} \mathrm{O}$ the geometry has been optimized in $\mathrm{C}_{2 \mathrm{v}}$ for the singlet $\mathrm{A}_{1}$ ground state $\left(d(\mathrm{O}-\mathrm{H})=0.95837 \AA, a(\mathrm{H}-\mathrm{O}-\mathrm{H})=105.6778^{\circ}\right)$ and the Löwdin function has been 
calculated using this geometry and $F C I / S T O-3 G$ singlet wave functions of $\mathrm{A}_{1}$ symmetry generated using the $G U G A$ algorithm $(N(F C I)=70 C S F$ 's $)$ using the $H F$ orbitals. We proceed similarly for $\mathrm{SH}_{2}\left(\mathrm{~A}_{1}\right.$ ground state in $\mathrm{C}_{2 \mathrm{v}}$ symmetry with $d(\mathrm{~S}-\mathrm{H})=1.33088 \AA$, $\left.a(\mathrm{H}-\mathrm{S}-\mathrm{H})=92.9297^{\circ} ; N(F C I)=382 C S F^{\prime} \mathrm{s}\right), \mathrm{CH}_{4}$ (singlet $\mathrm{A}_{1}$ ground state optimized in $\mathrm{C}_{2 \mathrm{v}}\left(d(\mathrm{C}-\mathrm{H})=1.08527 \AA, a(\mathrm{H}-\mathrm{C}-\mathrm{H})=109.4712^{\circ} ; N(F C I)=1436 C S F^{\prime} \mathrm{s}\right), \mathrm{NH}_{3}$ (singlet $\mathrm{A}^{\prime}$ ground state optimized in $\mathrm{C}_{\mathrm{s}}\left(d(\mathrm{~N}-\mathrm{H})=1.00967 \AA ⿻\right.$, $a(\mathrm{H}-\mathrm{N}-\mathrm{H})=107.9010^{\circ} ; N(F C I)=$ $\left.616 C S F^{\prime} \mathrm{s}\right), \mathrm{NH}_{4}^{+}\left(\right.$singlet $\mathrm{A}_{1}$ ground state optimized in $\mathrm{C}_{2 \mathrm{v}}(d(\mathrm{~N}-\mathrm{H})=1.01972 \AA, a(\mathrm{H}-$ $\left.\mathrm{N}-\mathrm{H})=105.6778^{\circ} ; N(F C I)=1436 C S F^{\prime} \mathrm{s}\right)$.

We also included two molecules having a triplet ground state: $\mathrm{CH}_{2}$ (triplet $\mathrm{B}_{1}$ ground state optimized in $\mathrm{C}_{2 \mathrm{v}}\left(d(\mathrm{C}-\mathrm{H})=1.07593 \AA\right.$, $a(\mathrm{H}-\mathrm{C}-\mathrm{H})=132.3511^{\circ}$; Löwdin function calculated using this geometry and $F C I / S T O-3 G$ triplet wave functions of $\mathrm{B}_{1}$ symmetry with $N(F C I)=148 C S F$ 's $)$ and $\mathrm{O}_{2}\left(\mathrm{~B}_{1 \mathrm{~g}}\right.$ ground state optimized in $\mathrm{D}_{2 \mathrm{~h}}(d(\mathrm{O}$ $\mathrm{O})=1.2215 \AA$ ); Löwdin function calculated using this geometry and FCI/STO-3G singlet wave functions of $\mathrm{B}_{1 \mathrm{~g}}$ symmetry with $\left.N(F C I)=106 C S F^{\prime} \mathrm{s}\right)$.

In Table 1 we report a set of WFT and DFT energy values for the set of simple molecules described above for which the Löwdin function defined in Eq. (9) has been constructed using the FCI results in a STO-3G minimal basis set. Here we report the energy of the systems (same $n$, same external potential $v\left(\mathbf{r}_{i} ; \mathbf{R}_{1} ; \cdots ; \mathbf{R}_{N}\right.$ ), and same basis set) obtained using $H F$ and several standard parameterizations of the $E_{X C}[\rho]$ functional for $L D A$ [22, 23], GGA (PW91PW91 [24], BLYP [25, 26], and PBEPBE [27]), metaGGA (revTPSS [28], PKZBPKZB [29], and VSXC [30]) and hybrid (B3LYP [26, 31] and $P B E O$ [32]). It should be emphasized that exactly the same FCI energies are obtained using different orbitals to construct the FCI space as expected from the fact that the same atomic basis set is used in all the cases.

In Table 2, we provide some relevant values for the Löwdin functions constructed from the FCI matrix using different sets of orbitals to analyze the dependence of the $H_{11}$ and the $f(0)$ values on the set of basis fuctions used to solve the FCI problem. In particular, we have used the $R H F / R O H F$ set of orbitals (orbital basis set A), symmetrized Huckel orbitals (orbital basis set B), the Kohn-Sham orbitals from $S$-VWN [22, 23] LDA functional (orbital basis set C), BLYP [25, 26] GGA functional (orbital basis set D) and the PBEO [32] hybrid functional (orbital basis set E). Finally, we also used the natural orbitals corresponding to the $F C I$ ground state to show the 
relevance of the exact one electron density matrix in defining the wave function of the ground state (orbital basis set F).

In all cases, the Löwdin function $f(E)$ for the present systems has the general structure shown in Figure 1 with the corresponding value of $K$ (dimension of the $F C I$ matrix). For the minimal basis set, all electronic states are bound (i.e.: $E_{i}<0$ for $i=1$, $K$ with $K=N(F C I)$ ) and, hence, the value $f(0)$ appears after the last vertical asymptote $\left(a_{K}\right)$. However, for a general case the $f(0)$ value can be located between two vertical asymptotes $a_{i}$ and $a_{i+1}$. This fact does not have any relevant consequence for the discussion below. We note that each vertical asymptote $a_{i}$ is an eigenvalue of the $\mathbf{H}_{I I, I I}$ matrix. In the present case we have only $K-1$ vertical asymptotes because this is the dimension of the $\mathbf{H}_{I I I I}$ matrix if the dimension of the full $\mathbf{H}$ is $K=N(F C I)$. When the $E$ variable takes a value $E=a_{i}$ then $f(E)$ function goes to infinity at this point. The $f(E)$ function is a non-increasing function of $E$. Finally we recall that as $E$ tends to $\pm \infty$ then $f$ (E) goes to $\mathbf{H}_{I, I}=H_{I l}=E_{\text {ref. }}$. In this case, each branch located within two consecutive vertical asymptotes, lets say, $a_{i}$ and $a_{i+1}$, cuts the straight line $f(E)=E$ one time and only one. This straight line passes through the point $(0,0)$. The point where a branch cuts the straight line is an eigenvalue of the full $\mathbf{H}$ matrix. The last branch cuts the straight line $f(E)=E$ at the point $E=E_{K}$ corresponding to the highest eigenvalue of this matrix, and also cuts the vertical axis $f(0)$ and from this point it goes to $E_{\text {ref }}$ as $E$ tends to $+\infty$. The $f(\mathrm{E})$ function when $E$ tends to $\pm \infty$ takes the values $E_{r e f}$ (which corresponds to $E_{H F}$ when the $H F$ orbitals are used (orbital set A), and to the expectation value of the Hamiltonian using the Huckel determinant, the $K S$ determinant or the natural determinant for the ground state (orbital sets B to F)). In other words, $\lim _{E \rightarrow+\infty} f(E)=\mathbf{H}_{I, I}$ $=H_{11}=E_{r e f}$. All these features show that the overall behavior of the calculated Löwdin function throughout the whole $E$ horizontal axis is well defined.

Regarding the particular values of the energies for the systems reported in Table 1 , we emphasize that for each system the same number of electrons $n$, the same external potential $v\left(\mathbf{r}_{i} ; \mathbf{R}_{1} ; \cdots ; \mathbf{R}_{N}\right)$, and same basis set has been used to obtain the energy using the FCI, HF and several standard parameterizations of the $E_{X C}[\rho]$ functional. For all the systems described above, the $H F$ energy is always above the exact $F C I$ energy, as known from the variational nature of the wave function expressed in terms of $C I$ expansions. In general, the DFT energies obtained using the selected functionals show 
important differences depending on the particular form of the exchange-correlation functional considered. The $L D A$ approach provides a value of the energy of the ground state that is above the exact FCI energy value. A detailed analysis of the values reported in Table 2 shows that the Löwdin function has essentially the same form but shows a the dependence of the $H_{11}$ and the $f(0)$ values on the set of basis fuctions used to solve the $F C I$ problem. The expected values of the energy $H_{11}=E_{\text {ref }}$ using the sets of orbitals $\mathrm{C}$ to $\mathrm{F}$ are slightly above the $H F$ energy, whereas a larger difference is observed when using the symmetrized Huckel orbitals. This is in line with the fact that the $H F$ determinant provides the lowest variational energy using a single determinant description. Interestingly enough, the $L D A$ energy value is also above the $H F$ energy and close to the value of the branch of the Löwdin function that cuts the $f(E)$ axis at $E=$ 0 . However, the GGA, meta-GGA and hybrid DFT energy values are all below the exact FCI value. As a general rule, the differences between energy values provided by the $G G A$, meta-GGA and hybrid DFT functionals are system dependent and larger for systems with larger number of electrons. A similar picture of the Löwdin function is obtained using different basis sets with a small variation of the $f(0)$ value (except perhaps when using the roughly approximated symmetrized Huckel basis set). Also, a significant change of the weight of the reference determinant in the ground state is observed for the differents orbital sets, with the larger value obtained using the natural orbitals of the FCI ground state. This value provides an estimation of the contribution of the one electron density matrix to the $F C I$ wave function.

In view of the previous discussion, the equivalence between $E[\rho]$ and the expression of the Löwdin function at $E=0$ provides an argument to explain this dispersion of results in terms of the complexity of the particular functional (larger number of parameters leads to lower energy) and the lack of variationality with respect to $E$. This comparison model suggests that the present DFT approaches require a revision to include an additional variational term in addition to the density $\rho(\mathbf{r})$, playing the role that the variable $E$ plays in the Löwdin function. From this comparison scheme it is clear that the variable $E$ controls the variational requirements in order to achieve a consistent description of the molecular electronic structure of both ground and excited states. The lack of an explicit dependence on $E$ in current DFT based approaches (along with the difficulties to treat open shell states) explains the limitations of this theory to describe excited states. Finally, this formal mathematical correspondence also suggests 
that $D F T$ functionals can be seen as effective single determinant approaches in the sense used by Clementi and coworkers to define their semi-empirical density functional approximations to obtain correlation energies from $H F$ wave-functions [33].

\section{Conclusions}

The results exposed in the previous sections reveals that the Löwdin function of Eq. (9) provides a tool of comparison model between the wave function and the density functional theories. This model provides an explicit form of the exact energy functional for a fermion system and reveals the inherent structure of both theories in order to be compared. The DFT functionals can be seen as special cases of Löwdin function that do not satisfy all necessary and sufficient conditions on $\rho(\mathbf{r})$. The latter implies that, if we are given any density function $\rho(\mathbf{r})$ which are not correctly derivable from a spin and space symmetry adapted wave function, there will always exist at least a functional which is capable of detecting this fact, in the sense that $\rho(\mathbf{r})$ will yield to an energy for this functional which is lower than its minimum energy. Ignoring the type of restrictions imposed by the spin and space symmetry adapted when making a variational calculation on such a system leads to an energy minimum that will exhibit the phenomenon of "overcorrelation". That is, the correlations expressed by the $\rho(\mathbf{r})$ function will be inconsistent with a $\gamma_{1}\left(\mathbf{r}_{1}, \mathbf{r}_{1}{ }^{\prime}\right)$ function derivable from a spin and space symmetry adapted wave function $\Psi\left(\mathbf{r}_{1} s_{1}, \cdots, \mathbf{r}_{n} s_{n}\right)$, even for a closed-shell system.

From this analysis, the increasing empiricism in defining current $E_{X C}$ based on the statistical performance is clearly limited by the form of the exact energy functional. Extension of DFT to include the dependence with $E$ to restore variationality may improve existent functionals to describe the ground and excited states.

\section{Acknowledgements}

Financial support has been provided by the Spanish Ministerio de Economía y Competitividad (formerly Ministerio de Ciencia e Innovación) through grants CTQ2011-22505 and FIS2008-02238) and by the Generalitat de Catalunya through grants 2009SGR-1472, 2009SGR-1041 and XRQTC. Part of the computational time 
has been provided by the Centre de Supercomputació de Catalunya (CESCA) which is also gratefully acknowledged. 
Figure 1. Qualitative representation of the Löwdin function $f(E)$ for a $F C I$ problem with $K=8 C S F^{\prime}$ 's and $H_{11}=E_{\text {ref }}$, to show the general behaviour of this function. In this example, the horizontal assimptote corresponds to $E_{\text {ref }}$ (i.e. single configuration representations: $R H F$ for closed shell systems, $R O H F$ or $G V B$ for open shell singlets and triplets). The points $E_{1} \leq E_{2} \leq E_{3} \leq \ldots \leq E_{8}$ correspond to the set of eigenvalues of the full $\mathbf{H}$ matrix of dimension $K=8$ (i.e.: those in which $f(E)=E$ ). The $K-1=7$ vertical assimptotes $a_{1} \leq a_{2} \leq a_{3} \leq \ldots \leq a_{7}$ correspond to the eigenvalues of the $K-1$ reduced $\mathbf{H}$ matrix, namely, $\mathbf{H}_{I I I I}$. See text for more explanation details of this plot.

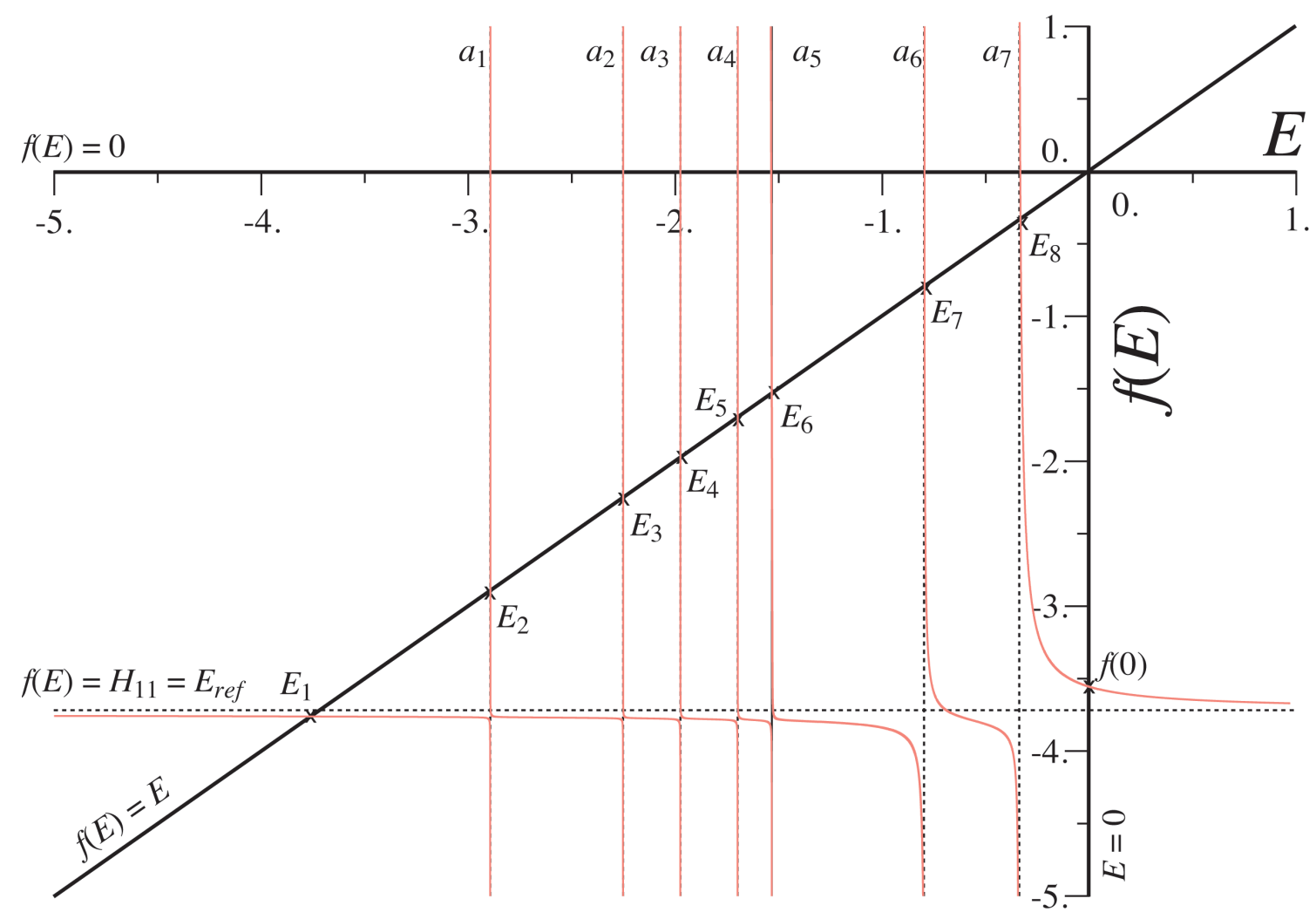


Table 1. Relevant energy values for the $F C I$ energies and energies of representative $E_{X C}[\rho]$ functionals. In all cases, the basis set is $S T O-3 G$ and the geometries correspond to $C I S D / 6-31++g^{* *}$ optimised structures (see text for details). The same sets of FCI eigenvalues are obtained independently of the orbitals used to construct the determinants discussed in Table II.

\begin{tabular}{|c|c|c|c|c|c|c|c|}
\hline & $\mathrm{H}_{2} \mathrm{O}$ & $\mathrm{NH}_{3}$ & $\mathrm{SH}_{2}$ & $\mathrm{CH}_{4}$ & $\mathrm{NH}_{4}{ }^{+}$ & $\mathrm{CH}_{2}$ & $\mathbf{O}_{2}$ \\
\hline$K=N(F C I)$ & 70 & 616 & 382 & 1436 & 1436 & 148 & 106 \\
\hline$E_{1}$ & -75.012114 & -55.517543 & -394.354092 & -39.805451 & -55.946702 & -38.472608 & -147.747895 \\
\hline$E_{2}$ & -74.419386 & -54.989548 & -393.780008 & -38.973091 & -55.099684 & -37.943644 & -147.146834 \\
\hline$E_{3}$ & -74.015740 & -54.931878 & -393.610959 & -38.917079 & -55.065056 & -37.888937 & -146.901982 \\
\hline$\ddot{E}_{K-1}$ & -28.052661 & -16.773511 & -179.589921 & -9.0789790 & -13.342182 & -11.697235 & -103.312082 \\
\hline$E_{K}$ & -27.401570 & -16.607329 & -177.750980 & -8.9003961 & -13.139050 & -11.366848 & -101.543057 \\
\hline$E(\mathrm{HF} / \mathrm{ROHF})$ & -74.962674 & -55.453330 & -394.311615 & -39.726846 & -55.866554 & -38.429892 & -147.632382 \\
\hline E(SVWN5) & -74.731653 & -55.289280 & -393.511068 & -39.616730 & -55.709647 & -38.235051 & -147.197578 \\
\hline E(PW91PW91) & -75.278919 & -55.752563 & -394.908835 & -40.006167 & -56.167937 & -38.617965 & -148.238832 \\
\hline$E(B L Y P)$ & -75.277026 & -55.744244 & -394.899925 & -39.994248 & -56.157526 & -38.614300 & -148.245053 \\
\hline Е(РВEРBE) & -75.225100 & -55.706750 & -394.755311 & -39.966679 & -56.123036 & -38.581657 & -148.141707 \\
\hline E(revTPSS) & -75.326254 & -55.796958 & -394.970071 & -40.051700 & -56.210114 & -38.666296 & -148.328874 \\
\hline E(PKZBPKZB) & -75.193024 & -55.688394 & -394.529979 & -39.958448 & -56.104226 & -38.573009 & -148.079450 \\
\hline E(VSXC) & -75.349301 & -55.814583 & -395.109922 & -40.059908 & -56.228813 & -38.668603 & -148.359499 \\
\hline E(PBE0) & -75.245260 & -55.725543 & -394.816928 & -39.984130 & -56.141393 & -38.599394 & -148.155159 \\
\hline E(B3LYP) & -75.312120 & -55.784317 & -394.951857 & -40.038517 & -56.197663 & -38.646414 & -148.273295 \\
\hline
\end{tabular}

